\title{
Observations of Diffuse and Translucent Clouds
}

\author{
PHILIPPE CRANE \\ European Southern Observatory \\ Garching. Germany
}

October 11. 1991

\begin{abstract}
Observations of interstellar $\mathrm{CN}, \mathrm{CH}$. and $\mathrm{CH}^{+}$in diffuse and translucent clouds obtained with a spectral resolution of more the 500,000 are reported. First results from a survey of $\mathrm{CH}$ and $\mathrm{CH}^{+}$are discussed. The $\mathrm{CH}^{+}$isotopic ratio has been determined to be $68 \pm 5$ toward $\zeta$ Oph and a component of ${ }^{12} \mathrm{CH}^{+}$has been found with a velocity width of $15 \mathrm{~km} \mathrm{~s}^{-1}$. A review of the rotational excitation temperature of interstellar CN and its implications is given.
\end{abstract}

\section{Introduction}

Advances in optical techniques in the last decade have provided a new dimension for the study of molecular species in diffuse and translucont clouds. Improved detectors have provided the means to detect weak lines, or to study stronger lines with vastly improved dynamic range. Advances in spec:trograph design and implementation have combined with the improved detectors to permit studies at vastly increased spectral resolution.

These advances have begun to yield new insights into the physical conditions and dynamics of diffuse and interstellar clouds. For the first time(1), interstellar absorption lines have been observed with roughly the same velocity resolution as the millimeter radio observations. These result are discussed in section 2 . where the first results of a survey at very high spectral resolution are mentioned.

Section 3 presents results for the $\mathrm{CH}^{+}$isotopic ratio. Section 4 presents some data on the excitation temperature of interstellar CN.

The visible interstellar absorption features discussed here are those of CN $\mathrm{R}(0)$ at $3874.607 \AA$. of $\mathrm{CH}^{+} \mathrm{R}(0)$ at $4232.548 \AA$ and of $\mathrm{CH} \mathrm{R}_{2}(1)$ at 4300.313A. For CN, in addition to the $R(0)$ line, the $R(1)$ and $P(1)$ lines in this system are also considered. The ${ }^{13} \mathrm{CH}^{+}$isotopic analog of ${ }^{12} \mathrm{CH}^{+}$is at $4232.267 \AA$.

\section{Ultra High Resolution Observations}

Optical observations with a spectral resolution of 500.000 or more are difficult not only because the spectrometers are not often capable of this resolution, but even when they are. such high resolutions require relatively bright stars. The work reported here has been performed at the Coude feed of the $2.7 \mathrm{~m}$ telescope of the MacDonald Observatory of the University of Texas. 
A first set of observations toward the star $\zeta$ Oph have been reported(1) for the $\mathrm{CN}, \mathrm{CH}$ and $\mathrm{CH}^{+}$. Following the success of these observations, a survey about 20 sight lines was started to study the $\mathrm{CH}$ and $\mathrm{CH}^{+}$lines.

\section{1. $\zeta$ OPH RESULTS}

Figure 1 shows a summary of the data obtained toward $\zeta$ Oph for the $\mathrm{CN}$, $\mathrm{CH}$, and $\mathrm{CH}^{+}$lines. These data have an effective resolution of $\lambda / \Delta \lambda=$ 600,000 . The CN line can clearly be seen to have two velocity components with a velocity separation of $1.18 \mathrm{~km} \mathrm{~s}^{-1}$. This coincides very closely with the velocity separation seen in the $\mathrm{CO}$ line profiles $(2 ; 3) \mathrm{The}^{+} \mathrm{CH}^{+}$line in these data consists of a single broad component with a velocity width of $\mathrm{b}=2.1 \mathrm{~km} \mathrm{~s}^{-1}$. A major surprise in these data is that the $\mathrm{CH}$ line shows elements of both the narrow features seen in the CN data and of the broad feature seen in the $\mathrm{CH}^{+}$line. This clearly demonstrates that $\mathrm{CH}$ exists in two different regions and under quite different physical conditions.

\subsection{SURVEY OF $\mathrm{CH}$ AND $\mathrm{CH}^{+}$}

Following the observations toward $\zeta \mathrm{Oph}$, a more comprehensive survey of other sight lines was undertaken. However, partly due to instrumental limitations, this survey, which is still in progress, was restricted to the $\mathrm{CH}$ and $\mathrm{CH}^{+}$species.

Table 1 provides a list of the sight lines which are being studied, and a very preliminary estimate of the line widths(FWHM) and relative velocities. The relative velocities given are the velocities of the sub-components relative to the strongest component, and not the relative velocity of $\mathrm{CH}$ relative to $\mathrm{CH}^{+}$. A separate observing program is underway to determine this.

One interesting result emerging from this survey is the relative frequency of multiple features in the $\mathrm{CH}$ lines but not in the corresponding $\mathrm{CH}^{+}$lines.

\section{High Signal-to-Noise Observations}

In order to further investigate a reported discrepancy $(4 ; 5)$ in the ${ }^{12} \mathrm{C} /{ }^{13} \mathrm{C}$ isotope ratio toward $\zeta \mathrm{Oph}$ as determined from $\mathrm{CH}^{+}$observations, several spectra were obtained of the $\mathrm{CH}^{+}$line at $4232.548 \AA$. The resulting spectrum is shown in Figure 2. In addition to finally providing close to a definitive result on the isotope $\operatorname{ratio}\left({ }^{12} \mathrm{C} /{ }^{13} \mathrm{C}=68 \pm 5\right)$, this spectrum also revealed a very broad feature in the ${ }^{12} \mathrm{CH}^{+}$feature. This broad feature has a width of about $15 \mathrm{Km} \mathrm{s}^{-1}$ FWHM and contributes $1.9 \mathrm{~m} \AA$ of equivalent width to the line whose total equivalent width is $23.25 \mathrm{~m} \AA$. 


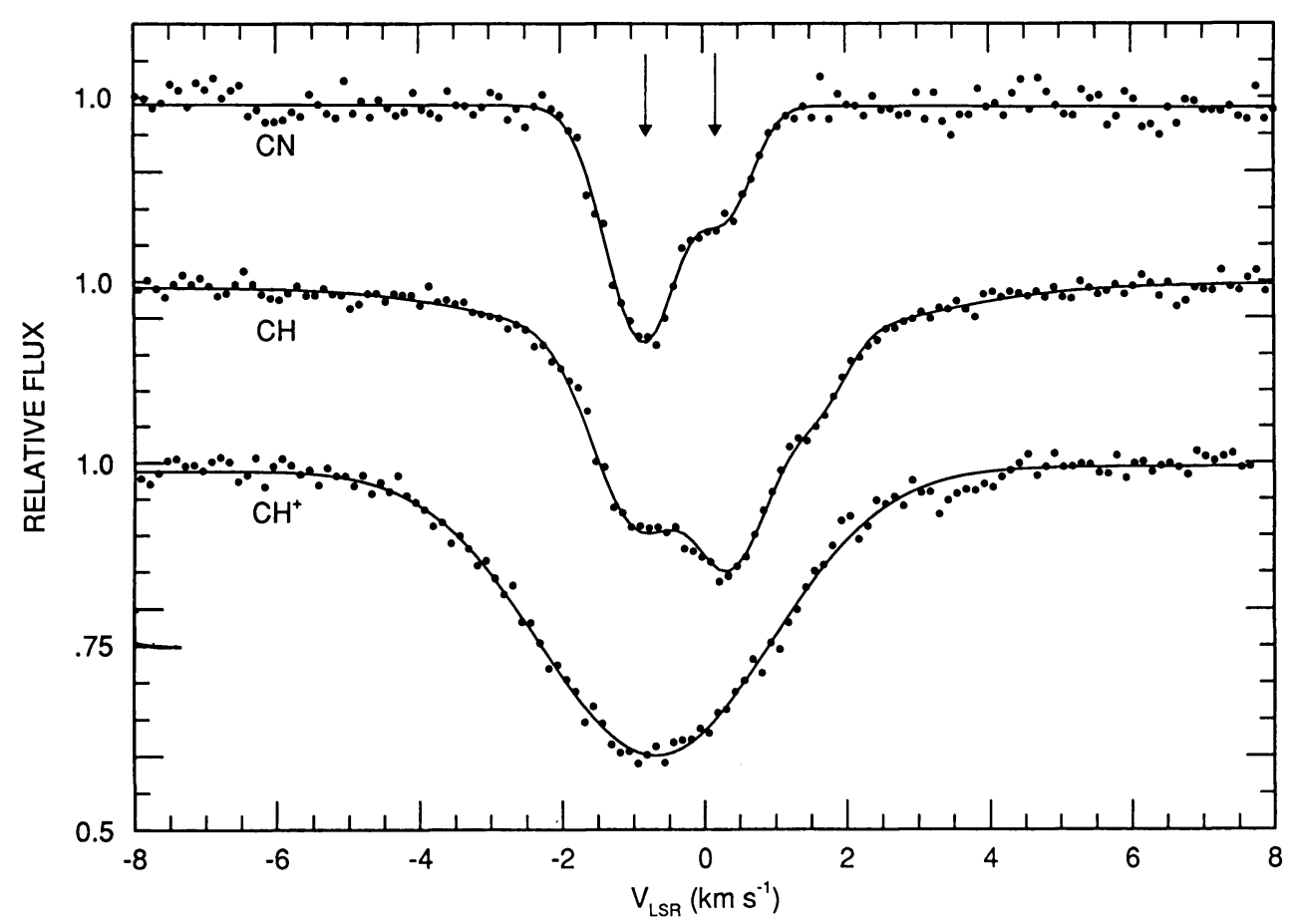

Fig. 1. Plot of CN lines toward $\zeta$ Oph. The top spectrum shows the CN line, and indicates the positions of the two velocity components. The middle spectrum shows the $\mathrm{CH}$ line, where the effects of the lambda doubling are evident, as well as the effects of a broad component underlying the narrower components. The bottom spectrum shows the $\mathrm{CH}^{+}$line which consists of a single Gaussian.

\section{CN Excitation Temperature}

The excitation temperature of the lowest rotational levels of interstellar CN has proved to be a good means for determining the temperature of the Cosmic Background Radiation(6). However. after the launch of the COBE satellite(7) and the successful rocket experiment of Gush, Halpern. and Wishnow (8), there appears to be a possible discrepancy between the CBR temperature determined by the space experiments, and the best determinations of the CN excitation temperature(9). It has been proposed(10) that the origin of this difference could be local processes in the interstellar clouds which could excite the CN rotational levels a few millikelvin above the CBR.

In an attempt to test the local excitation hypothesis, Palazzi. Mandolesi, and Crane(9) have collected data from several new sight lines as well as from sources in the literature. By using the primary data equivalent widths, and 
TABLE I

Preliminary Survey Results

\begin{tabular}{|c|c|c|c|c|c|c|}
\hline & \multicolumn{3}{|c|}{ CH Data } & \multicolumn{3}{|c|}{$\mathrm{CH}^{+}$Data } \\
\hline Star & $\mathrm{v}_{r \epsilon l}$ & $\mathrm{~W}_{\lambda}(\mathrm{m} \AA)$ & $\sigma(\mathrm{m} \ddot{A})$ & $\mathrm{v}_{r \epsilon l}$ & $\mathrm{~W}_{\lambda}(\mathrm{m} \dot{A})$ & $\sigma(\mathrm{m} \AA)$ \\
\hline 23 Ori & & & & 0.00 & 3.90 & 26.14 \\
\hline 67 Oph & 0.00 & 0.88 & 15.43 & 0.00 & 1.33 & 21.60 \\
\hline & 1.44 & 0.176 & 12.11 & & & \\
\hline$\kappa \mathrm{Aql}$ & 0.00 & 1.38 & 10.57 & & & \\
\hline 68 Cyg & 0.00 & 1.27 & 10.94 & & & \\
\hline & -0.254 & 1.25 & 31.67 & & & \\
\hline$\lambda$ Cep & 0.00 & 14.57 & 6.85 & & & \\
\hline & 0.418 & 3.88 & 24.95 & & & \\
\hline$\mu \mathrm{Sgr}$ & 0.00 & 1.54 & 14.38 & & & \\
\hline & -1.422 & 0.28 & 18.51 & & & \\
\hline$\rho \mathrm{OphA}$ & 0.00 & 2.23 & 6.51 & & & \\
\hline & 0.00 & 2.06 & 18.80 & & & \\
\hline$\psi$ Per & 0.00 & 1.68 & 11.21 & 0.00 & 3.51 & 17.27 \\
\hline & 1.19 & 1.41 & 12.04 & 1.14 & 4.25 & 16.72 \\
\hline & 2.09 & 0.91 & 54.69 & 2.22 & 0.74 & 30.83 \\
\hline$\zeta$ Per & 0.00 & 2.92 & 8.80 & 0.00 & 0.86 & 18.37 \\
\hline & -0.28 & 3.01 & 20.22 & & & \\
\hline o Per & & & & 0.00 & 1.99 & 21.38 \\
\hline$\chi \mathrm{Oph}$ & 0.00 & 7.53 & 14.49 & 0.00 & 4.14 & 18.61 \\
\hline$\delta$ Sco & 0.00 & 0.00 & 7.00 & 0.00 & 0.40 & 12.03 \\
\hline & 0.48 & 0.26 & 10.81 & & & \\
\hline$\beta^{1}$ Sco & 0.00 & 0.81 & 24.06 & & & \\
\hline 20 Tau & & & & 0.00 & 8.96 & 18.47 \\
\hline $23 \mathrm{Tau}$ & & & & 0.00 & 5.81 & 20.09 \\
\hline
\end{tabular}

b values, they have produced a homogeneous set of $\mathrm{CN}$ excitation temperatures. They find a weighted mean $\mathrm{CN}$ excitation temperature of $2.817 \pm 0.022$ $\mathrm{K}$ which is about $82 \mathrm{mK}$ above the space values. They have searched their data set for correlations of the excitation temperatures with a wide range of parameters. There is no correlation with electron density, distance from the Sun, or with any other plausible parameter. More observations will be necessary to confirm the excitation temperature discrepancy, or to understand its origin.

\section{Acknowledgements}

Much of the work presented here has been done in collaboration with David Lambert, Reno Mandolesi, and Eliana Palazzi. It is a pleasure to acknowledge their contributions to this work. 


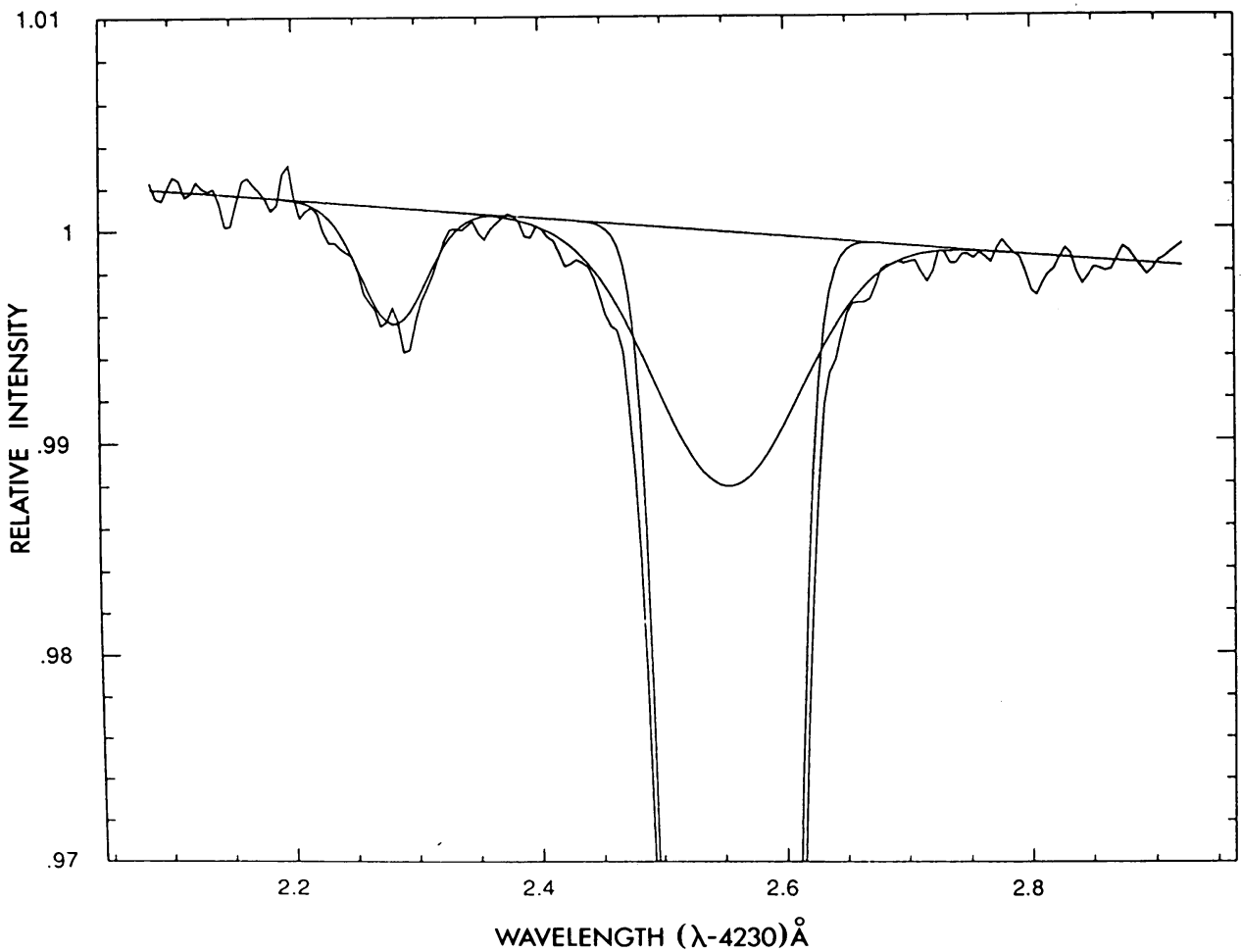

Fig. 2. High signal-to-noise data for $\mathrm{CH}^{+}$toward $\zeta \mathrm{Oph}$. The depth of the stronger feature reaches a relative intensity of 0.67 . The ${ }^{13} \mathrm{CH}^{+}$line is clearly visible on the left of the strong ${ }^{12} \mathrm{CH}^{+}$line. The solid line indicates the subcomponents used to fit the data, and illustrates the presence of the very broad component.

\section{References}

1) Lambert.D.L., Sheffer.Y., and Crane,P., $C N, C H$, and $\mathrm{CH}^{+}$Toward $\zeta$ Ophiuchi, Astrophysical Journal (Letters),359,L19, 1990.

2) Lauger.W.D., Glassgold,A.E., and Wilson,R.W., 1987, Radio Observations of Carbon Monoxide toward Zeta Ophiuchi: Velocity Structure. Isotopic Abundances, and Physical Properties Astrophysical Journal.322.450.

3) Crutcher,R.M.. and Federman,S.R.,1987, The CO J=2-1 Emission from the Interstellar Gas toward Zeta Ophiuchi. Astrophysical Journal (Letters).316,L71.

4) Hawkins.I.. and Jura,M., 1987, The ${ }^{12} C /{ }^{13} C$ Ratio of the Interstellar Medium in the Neighborhood of the Sun. Astrophysical Journal.317,926.

5) Stahl.O., Wilson.T.L., Henkel,C., and Appenzeller,I., $1989 \mathrm{CH}^{+}$toward Zeta Oph Astronomy and Astrophysics,221, 321.

6) Cranc.P.. Hegyi.D.J., Kutner,M.L., and Mandolesi,N., Cosmic Background Radiation Temperature at 2.64 Millimeters Astrophysical Journal,346.136, 1989

7) Mather,J., et al., A Preliminary Measurement of the Cosmic Microwave Background Spectrum by the COBE Satellite, Astrophysical Journal (Letters),354,L37, 1990.

8) Gush,H.P., Halpern,M., and Wishnow.E.H., Rocket Measurement of the Cosmic Background Radiation mm Wave Spectrum. Physical Review Letters,65,537, 1990. 
9) Palazzi,E., Mandolesi,N., and Crane,P., CN Rotational Excitation, Astrophysical Journal, submitted, 1992.

10) Black,J.H., and van Dishoeck,E.F., Electron Densities and the Excitation of $C N$ in Molecular Clouds, Astrophysical Journal ( Letters),369,L9, 1991.

\section{DISCUSSION}

N Wright:

$=$ Comment 1: The largest signal from the galaxy in the COBE $3 \mathrm{~mm}$ maps is $\leq 1 \mathrm{mK}$, so dust emission is unlikely to raise the $\mathrm{T}_{\text {exc }}(\mathrm{CN})$ above $\mathrm{T}_{C M B}$ $=$ Comment 2: The equivalent width of the ${ }^{12} \mathrm{CN} R(0)$ line in your high spectral resolution data is less than in your high SNR data, suggesting that CN also has a weak broad component.

$=$ Question: The line widths you quote are too large to be velocity dispersions. Do you mean FWHM?

- P. Crane: I agree with both your comments! The line widths quoted are indeed FWHMs and they are in $\mathrm{m} \AA$.

- M. Guelin: Is there in your CN excitation temperature a correlation of the excitation temperature with the error in determining the excitation temperature?

- P. Crane: There does not appear to be such a correlation.

- J.C. Pecker: This difficulty about the excitation temperature of CN reminds me of a very different case, that of the solar atmosphere. There. in spite of large temperatures and densities, we found (in the fifties!) a $\Delta \mathrm{T}_{\text {exc }}$ very large between the rotational levels and the electronic levels. We could not explain it because of lack of reliable collisional cross sections involving all relevant levels. In spite of the highly different conditions, this lack of physical data, could it be a reason for the unexplained $\Delta \mathrm{T}_{\epsilon x c}$ discrepancy? Or perhaps an underestimate of the number of transitions involved?

- P. Crane: I have never thought of this, although it seems at first glance unlikely, I will look more into your suggestion. 